\title{
How we are misinterpreting physical activity intention - behavior relations and what to do about it
}

\author{
Amanda L. Rebar ${ }^{1 *} \mathbb{D}$, Ryan E. Rhodes² and Benjamin Gardner ${ }^{3}$
}

\begin{abstract}
Background: Studies of the physical activity intention-behavior gap, and factors that may moderate the gap (e.g., habit, perceived behavioral control), can inform physical activity promotion efforts. Yet, these studies typically apply linear modeling procedures, and so conclusions rely on linearity and homoscedasticity assumptions, which may not hold.

Methods: We modelled and plotted physical activity intention-behavior associations and the moderation effects of habit using simulated data based on (a) normal distributions with no shared variance, (b) correlated parameters with normal distribution, and (c) realistically correlated and non-normally distributed parameters.

Results: In the uncorrelated and correlated normal distribution datasets, no violations were unmet, and the moderation effects applied across the entire data range. However, because in the realistic dataset, few people who engaged in physical activity behavior had low intention scores, the intention-behavior association was non-linear, resulting in inflated linear moderation estimations of habit. This finding was replicated when tested with intention-behavior moderation of perceived behavioral control.

Conclusions: Comparisons of the three scenarios illustrated how an identical correlation coefficient may mask different types of intention-behavior association and moderation effects. These findings highlight the risk of misinterpreting tests of the intention-behavior gap and its moderators for physical activity due to unfounded statistical assumptions. The previously well-documented moderating effects of habit, whereby the impact of intention on behavior weakens as habit strength increases, may be based on statistical byproducts of unmet model assumptions.
\end{abstract}

Keywords: Habit, Assumption testing, Motivation, Simulation, Exercise, Goals

Physical inactivity is the fourth leading risk factor for global mortality - leading to an estimated 3.2 million preventable early deaths [1]. Despite ongoing global efforts to promote physical activity [2], most of the population in developed countries remains either entirely inactive or insufficiently active to optimally benefit from the physical and mental health benefits of regular activity (e.g., $[3,4])$. There is a pressing need to understand how to effectively promote physical activity. Most individual-level interventions aim to promote physical activity through enhancing intentions - representations of self-instructions regarding either the

\footnotetext{
* Correspondence: a.rebar@cqu.edu.au

${ }^{1}$ School of Health, Medical, and Applied Sciences, Central Queensland University, Building 18, Room 1.33, Bruce Highway, Rockhampton, Queensland 4703, Australia

Full list of author information is available at the end of the article
}

direction of a goal (i.e., a goal to quit smoking), the intensity of commitment to act or not act (i.e., I strongly commit to my goal of quitting smoking), or a combination of both [5, 6].

Intention (in some form) is present in commonly applied theories to health behavior, including the theory of planned behavior [7], transtheoretical model [8], protection motivation theory [9], health action process approach [10], and social cognitive theory [11]. Typically, physical activity intentions are measured using continuous scales of the quantity of planned activity (i.e., decisional intention; 'I intend to engage in _ minutes of physical activity next week') or the degree of commitment a person has to enact their intention (i.e., intention strength; 'To what degree do you intend to engage in physical activity next week?': 'Very little - Very much') [5]. 
Intentions predict a substantial amount of variability of future physical activity duration and frequency [12-15]. In a meta-analysis of prospective correlational studies, McEachan et al. estimated that intentions explain $33 \%$ of variability in future physical activity behavior [14]. Experimental evidence also points to the importance of intention in predicting change in physical activity behavior, although to a smaller degree than seen in correlational studies [16, 17]. In their meta-analysis, Rhodes and Dickau found that experimental manipulations of activity intentions $(d=0.45)$ led only to small changes in physical activity $(d=0.15)[16]$. The consistent intentionbehavior link found in physical activity research suggests there is value in promoting intentions, but also illustrates that much variability in physical activity is not explained by intention. This disconnect has been coined the intentionbehavior gap $[15,18]$. Understanding behavior requires understanding not only intentions, but also factors that affect the likelihood of acting on intentions.

The process of translating intentions into behavior has been described as action control $[15,19]$. To understand what factors contribute to action control of health behaviors, researchers have investigated person, context, and state variables that may moderate the magnitude of intention-behavior relationships. Such variables have included characteristics of intention itself (e.g., intention stability) [20], and more conceptually distinct factors, such as moral norms [21], habit [22, 23], self-efficacy [24], planning [25], and executive functioning [26], amongst others [19].

Investigations into moderators of the impact of intention on future behavior have both theoretical and applied merit, but they typically use linear modeling. Linear models make assumptions about how data are structured that have not been adequately tested for physical activity intention-behavior relationships. Linear modeling can be misleading if there are digressions from these assumptions [27-29]. For example, a simple but essential assumption of linear regression is homoscedasticity - that there is constant variance across the range of residuals for each predictor variable, such that the variability left unexplained by the model is the same for people with low and high scores [27, 29]. Another major assumption is that relationships between the outcome variable and the predictor variables within the regression model are linear, in that direct associations hold true for people with high and low values of both variables. Violations of assumptions of homoscedasticity or linearity result in misleading estimates of the magnitude and statistical significance of regression coefficients [28]. Even if the true effect does withstand the assumption violations, there is a high likelihood the findings of such models may only hold for a specific sub-group of the sample, so not be fully representative of the target population $[27,29]$. In the real world, the distributions of physical activity intention and behavior data tend to be asymmetrical, resulting in non-normal model error distributions and non-linear relationships [19], so not meeting assumptions of linear modeling. Such methodological concerns are not trivial; seemingly small divergences in modelling assumptions can translate into misleading conclusions.

People tend to have intentions concordant with healthy action. For example, people who sign up for a physical activity study will likely have stronger activity engagement intentions than the general population. This sampling bias phenomenon oftentimes manifests as a skewed intention variable distribution, in which more scores than expected are higher than the mean (e.g., ceiling effect) [30]. Distributions of physical activity behavior frequency and duration variables are rarely symmetrical either. Oftentimes, such variables are positively or negatively skewed, sometimes to the extent that they are severely inflated (i.e., more zero scores than anticipated) [31], which can lead to skewed residual distributions when used in linear models.

Digression of physical activity intention and behavior data from linear modelling assumptions is a major concern for intention-behavior gap moderation testing. This is particularly so if a hypothesized moderator variable is correlated with intention and behavior (even to a modest degree), which can exacerbate the biases from these unmet assumptions [28]. Take, for example, studies of habit. Habit is the process by which a person's behavior is influenced from a prompt to act based on well-learned associations between cues and behaviors [32, 33]. As an automatic response to contextual cues, habit is expected to generate behavior in the presence of cues more rapidly and effectively than is intention, such that people are hypothesized to be more likely to act in line with habits than intentions [6, 32, 33]. Many studies have investigated whether habit could thus help explain the intention-behavior gap, such that people with weak intentions may nonetheless act where they have a strong habit for doing so [22, 34]. Most commonly, physical activity habit strength is assessed using self-reported scales reflecting the degree to which a behavior is experienced as being automatic (e.g., 'Physical activity is something I do automatically:' Strongly Disagree - Strongly Agree) $[35,36]$.

Gardner et al.'s review found that habit moderated intention-behavior associations in eight of nine available studies, such that intention-behavior associations decreased as habit strength increased [22]. Such findings are typically used to inform recommendations for promoting physical activity. Indeed, Gardner et al. concluded that "the failure of intention to translate into action where habit is strong suggests that motivation change ... will not change unhealthful habits" [22]. 
However, physical activity habit can be highly associated with behavior and intention [32, 37]; habit typically forms through repetition of an intended action [38], such that habitual tendencies will often concur with intentions. In this case, there may be few people for whom intention is weak, yet habit is strong [34]. Therefore, the interpretation of these intention-behavior moderation findings may be misleading.

The risk for misinterpretation of significant moderation effects on intention-behavior associations is not exclusive to habit, however. It applies to the common scenario in which 1 - intention and behavior have a non-linear relationship, and 2 - the moderator variable shares substantial variability with the behavior and/or intention variable. These circumstances apply to many investigated moderator effects in the study of physical activity [34, 39] and many other health behaviors [13, 14]. Perceived behavioral control, which reflects a person's assessment of the ease or difficulty in performing the behavior [7], provides another well-established intention-behavior moderation example. In its original conception, the theory of planned behavior postulated that perceived behavioral control would moderate intention-behavior associations, in that higher perceived control should lead to a better execution of intentions into behavior. The moderating effect of perceived behavioral control (or related constructs such as capacity) has been consistently replicated for physical activity [16] and other health behaviors [13, 14]. Metaanalyses show typically medium-sized associations between perceived behavioral control and a variety of health behavior outcomes $(r ' s=0.31,0.39)[13,14]$ and between perceived behavioral control and intention $(r ' s=0.54$, $0.60)[13,14]$. If intention-behavior associations are asymmetrical, this questions interpretations of the observation that perceived behavioral control statistically moderates intention and behavior associations. The robustness of tests of intention-behavior moderation warrant empirical investigation.

Simulated data can shed light on issues raised when methods and their tethered theoretical implications are questioned. Simulated studies apply algorithm-generated data distributions based on pseudo-random sampling from known probability distributions of extant evidence of the variables under study; in this way, data are hypothetical, yet informed by previous empirical evidence [40]. For example, Simmons et al. used simulated data to estimate that the probability of false-positive findings in psychology experiments is likely much higher than .05 , based on evidence of the breadth of flexibility of researchers' choices about study variables and analyses [41]. This, in part, led to re-evaluation not only of specific studies, but also of popular methodology within psychology, and the promotion of conceptual replications, use of Bayesian statistics, and pre-registration of study procedures as corrective measures to improve evidence quality and validity.

\section{The present study}

Our aim was to investigate how the interrelatedness and typical asymmetrical distributions of intention and behavior data may affect interpretations of moderators of intention-behavior associations, particularly moderators commonly aligned with intention and physical activity behavior, such as habit and perceived behavioral control. We used simulated data to compare three scenarios of physical activity intention-behavior associations and habit moderation. The comparison tested whether the common interrelatedness of these variables leads to misleading moderation conclusions. Then, we used simulated data of the same scenario with perceived behavioral control as a moderator of intention-behavior associations to demonstrate the generalizability of the findings across other moderator variables.

\section{Methods}

Three sets of physical activity intention, behavior, and habit data and one set of intention, behavior, and perceived behavioral control data were simulated using the MASS function in $R$ version 3.4.1 [42, 43] based on a priori set parameters with $N=100$ with bootstrapped estimates based on 7500 replications of the raw data $[44,45]$. The distribution and covariance parameters were determined based on effect sizes of meta-analyses of prospective associations [13, 14, 22, 34, 37]. All variables were set as continuous interval scales with standardized ranges. Histograms of the sampled means were visually inspected and no abnormalities were detected. All distributions were near Gaussian with very little skew $(<.40)$. The first set normal, unrelated - represented intention, behavior, and habit data with no shared variability, all normally distributed; this represents a perfect statistical model, in that it does not violate model assumptions by any degree. The second set - normal, correlated - met the homoscedasticity and linearity assumptions of linear modelling (i.e., normally distributed with linear relationship), but with correlated intention, behavior, and habit data to the magnitude found in previous meta-analyses. The third dataset - realistic - incorporated the same magnitude of correlations between intention, behavior, and habit as in the correlated set, but these were set to more closely mirror the non-normal distributions and, therefore, asymmetrical associations typical of intention-behavior relationships observed in previous physical activity research. For the test of generalizability, the intention and behavior variables were set to the same univariate parameters as in the realistic dataset (i.e., non-normal distributions) and the perceived behavioral control variable was set to be normally distributed, but the parameters of the correlations of 
perceived behavioral control with intention and physical activity behavior were set based on meta-analytic findings $[13,14]$. The study was exempt from needing ethical clearance, as the data were computer-generated.

\section{Univariate distribution parameters}

The normal, unrelated and normal, correlated simulation data distributions were set to be near Gaussian. For all models, the moderator variables of habit and perceived behavioral control were also set to be near Gaussian. For the realistic model and test of generalizability, the intention and behavior variable distributions were weighted based on the quartile proportions of intentionbehavior profiles as found in Rhodes and de Bruijn [34]. The intentions variable was negatively skewed, reflective of most people having high scores (skewness $=-0.70$, kurtosis $=3.18$ ) and the behavior score was flattened out, reflective of fewer people having mid-range scores than would be expected with normal distribution (skewness = 0.11 , kurtosis $=1.67$ ).

\section{Multivariate covariation parameters}

The normal, unrelated intention, behavior, and habit variables were all set to have null correlations $(r \sim 0.00)$. For the normal, correlated and realistic sets of simulations, the intention-behavior bivariate correlation was set at $r=0.49$, based on the meta-analytic findings of prospective intention-behavior correlational studies of physical activity $[13,46]$. Across both the normal, correlated and realistic datasets, habit was set to positively associate with both behavior $(r=.41)$ and intention $(r=.49)$. These association effect sizes were set a priori based on systematic review and meta-analytic findings of bivariate, direct associations [16, 22, 37]. For the test of generalizability, perceived behavioral control and intention were set to correlate at $r=.47$, and perceived behavioral control and behavior at $r=.33$, in line with meta-analytic findings [13, 14].

\section{Moderators of the intention-behavior relationship}

To test the moderating effect of habit and perceived behavioral control on intention-behavior associations, we estimated simple linear regression models with behavior predicted by mean-centered intention and moderator variables, as well as their interaction terms. The bootstrapped estimates are presented as well as the $S D$ of the bootstrap estimates and the interquartile ranges $(25,75 \%)$ of the replication estimates. For illustrative purposes, moderation effects were plotted with trend lines shown for people with high $(>M+1 S D)$, average $(<M+1 S D \&>M-1 S D)$, and low $(<M-1 S D)$ moderator scores.

\section{Results \\ Intention-behavior association \\ Normal, unrelated data}

Figure 1 shows the scatterplot and correlation line with +/- 2 standard error intervals of normal, unrelated intention-behavior data. Based on these simulations, 26\% of people had high intention but low behavior scores, $27 \%$ of people had high intention and high behavior scores, $22 \%$ had low intention and low behavior scores, and $25 \%$ had high intention and behavior scores. This near-equal distribution is what would be expected with a near perfectly normal distribution, resulting in a near null association of $r \sim 0.00$ (bootstrap $S D=0.09$ ).

\section{Normal, correlated data}

Figure 2 shows the scatterplot and correlation line with $+/-2$ standard error intervals of the simulated intention-behavior data with normal distributions and a correlation of $r=.49$ (bootstrap $S D=0.09$ ). Based on these simulations, there were only $16 \%$ of cases with high intention but low behavior scores. There were 28\% of cases with high intention and behavior scores, $34 \%$ with low intention and low behavior scores, and 22\% with low intention but high behavior scores. Within this fictitious scenario in which data are normal and linearly related, the intention-behavior gap is a result of both the people who made intentions and did not follow through with them $(16 \%)$ as well as the $22 \%$ of people who engaged in the behavior without intention.

\section{Realistic data}

Figure 3 shows the scatterplot and correlation line with $+/-2$ standard error intervals of simulated intention and behavior data with the same correlation of $r=.49$ (bootstrap $S D=.07$ ); however the distributions were corrected to reflect the reality of typical physical activity intention and behavior data distributions as found in Rhodes and de Bruijn [34]. Compared to the simulated data with normal distributions, this scenario shows far more cases with high intention and low behavior scores (30\%) and far fewer cases with low intention but high behavior scores $(4 \%)$.

\section{Habit as moderator of intention-behavior associations Normal, unrelated data}

Table 1 and Fig. 4 show a moderation effect of habit on the intention-behavior association amongst the normally distributed, unrelated dataset $(b=-0.17)$. Of the 7500 simulations, $50 \%$ of moderation effects fell within the range of $b=-0.24$ and -0.12 . For people with low habit scores, the association between intention and behavior was $b=0.72$ (dotted line); whereas the association was near null for people with either average $(b=-0.05$, solid line) or high ( $b=-0.09$, dashed line) habit scores. In this 


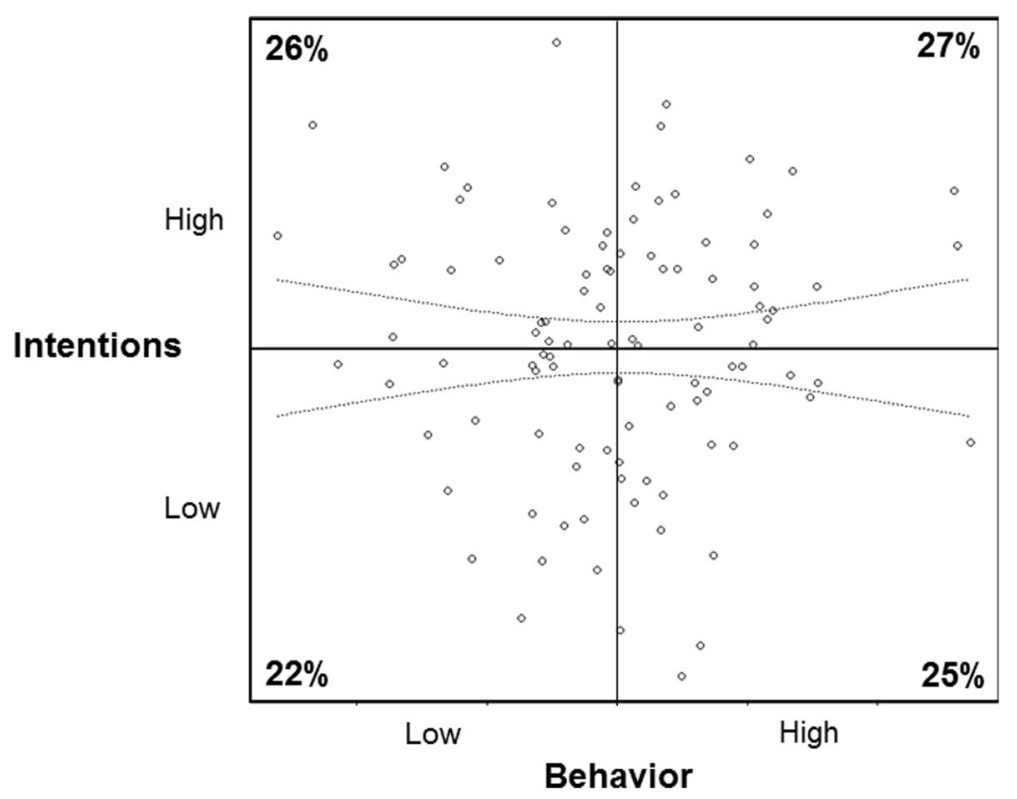

Fig. 1 Simulated normally distributed, unrelated intention and behavior data set to near Gaussian distributions with a correlation of $r \sim .00$

instance, effects would be interpreted as evidence that the intention-behavior correlation is stronger for people with weaker habits (i.e., smaller intention-behavior gaps) than people with average or stronger habits.

\section{Normal, correlated data}

Table 1 and Fig. 5 show the moderation effect of habit on the intention-behavior association amongst the normally distributed, correlated dataset $(b=0.01)$. Of the 7500 simulations, $50 \%$ of moderation effects fell within the range of $b=-0.05$ and 0.04 . Under these circumstances, people with low habit scores showed an intention-behavior association of $b=.51$ (dotted line), just slightly more steep than that of the association between intention and behavior for people with average ( $b=.44$; solid line) or high $(b=.30$; dashed line $)$ habit scores. For this example, the apparent moderation effect is such that there is a slightly stronger association between intention and behavior for people with low habit scores. Of note, because the intention-behavior variables

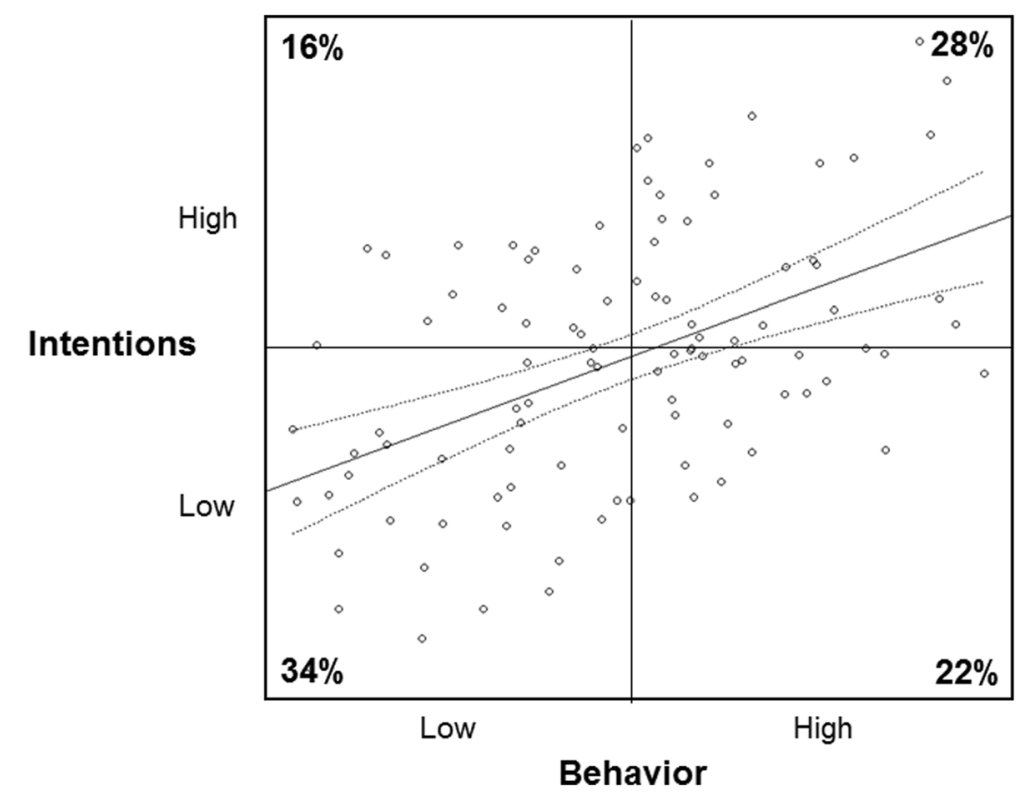

Fig. 2 Simulated normally distributed, correlated intention and behavior data set to near Gaussian distributions with a correlation of $r \sim .49$ 


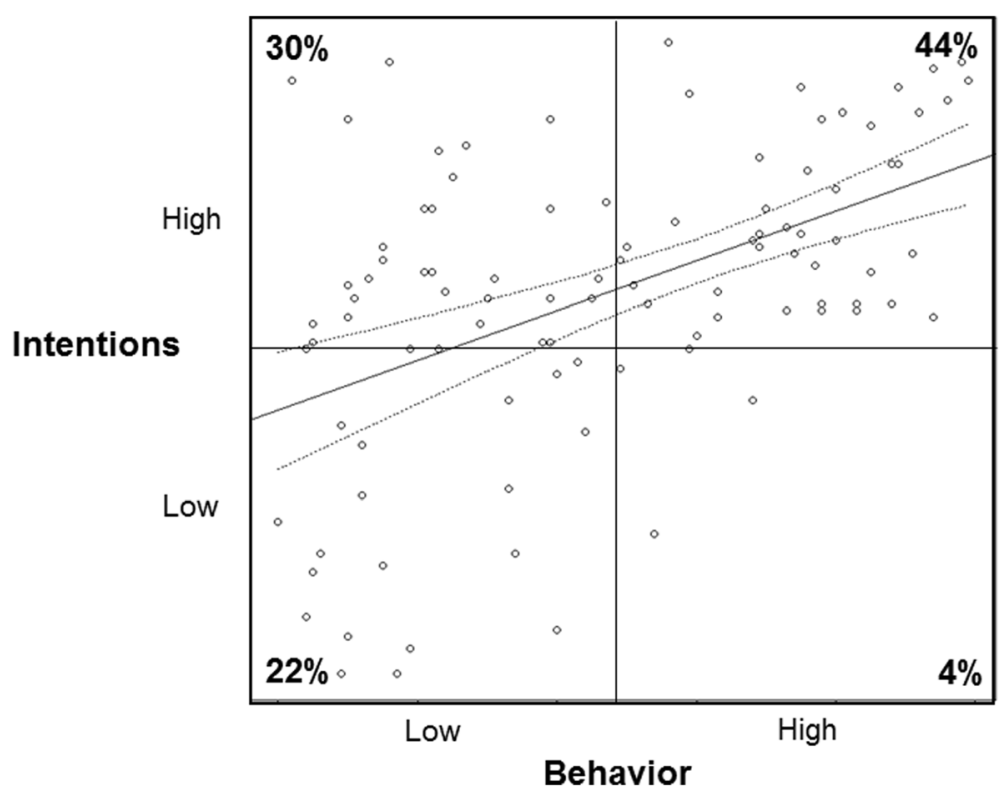

Fig. 3 Simulated realistic intention and behavior data with non-normal distributions commonly seen in health behavior research (intention negatively skewed, behavior positively skewed) and a correlation of $r \sim .49$

are correlated, the data range is mostly distributed in the top-right and bottom-left quadrants, such that the trend lines for habit moderation are restricted.

\section{Realistic data}

. Table 1 and Fig. 6 show the moderation effect of the simulated habit variable on the intention-behavior data with realistic correlations and distributions $(b=0.07)$. Of the 7500 simulations, $50 \%$ of moderation effects fell within the range of $b=-0.46$ and 0.69 . In this instance, for cases with low habit scores, the association between intention and behavior is $r=0.59$ (dotted line); whereas it is $r=0.50$ (solid line) and $r=0.40$ (dashed line) for cases with average and high habit scores, respectively. Of note, there are no cases of high habit in the bottom two quadrants (which represents people with low intention

Table 1 The Estimated Moderation Effects and Probing Analyses of Habit on the Intention-Behavior Association Applying 7500 Bootstrap Replications for Normally Distributed, Unrelated; Normally Distributed Correlated; and Realistic Intention-Behavior Data

\begin{tabular}{lllll}
\hline Data & Moderation effect of Habit, $b$ & Intention-Behavior, $b$ & Bootstrap SD & Interquartile Range of Bootstrap Estimates \\
\hline Normal, Unrelated & -0.17 & - & 0.10 & -0.24 to -0.12 \\
Low Habit & - & 0.72 & 0.34 & 0.55 to 0.96 \\
Average Habit & - & -0.05 & 0.13 & -0.14 to 0.03 \\
High Habit & - & -0.09 & 0.16 & -0.20 to 0.01 \\
Normal, Correlated & 0.01 & - & 0.07 & -0.05 to 0.04 \\
Low Habit & - & 0.51 & 0.24 & 0.36 to 0.65 \\
Average Habit & - & 0.44 & 0.10 & 0.37 to 0.50 \\
High Habit & - & 0.30 & 0.25 & 0.13 to 0.41 \\
Realistic & -.07 & - & 0.89 & -0.46 to 0.69 \\
Low Habit & - & 1.14 & 0.65 & 0.70 to 1.64 \\
Average Habit & - & 0.57 & 0.16 & 0.47 to 0.68 \\
High Habit & - & 0.58 & 0.15 & 0.48 to 0.67
\end{tabular}

Notes. Normal, unrelated: All variables set near Gaussian; intention-behavior $r \sim 0.00$; habit-behavior $r \sim 0.00$; habit-intention $r=.47$. Normal, correlated: All variables set near Gaussian; intention-behavior $r=0.49$; habit-behavior $r=0.41$; habit-intention $r=.47$. Realistic: intentions: skewness $=-0.70$, kurtosis $=3.18$; behavior: skewness $=0.11$, kurtosis $=1.67$; habit and perceived behavioral control set near Gaussian; intention-behavior $r=0.49$; habit-behavior $r=0.41$; habit-intention $r=.47$ 


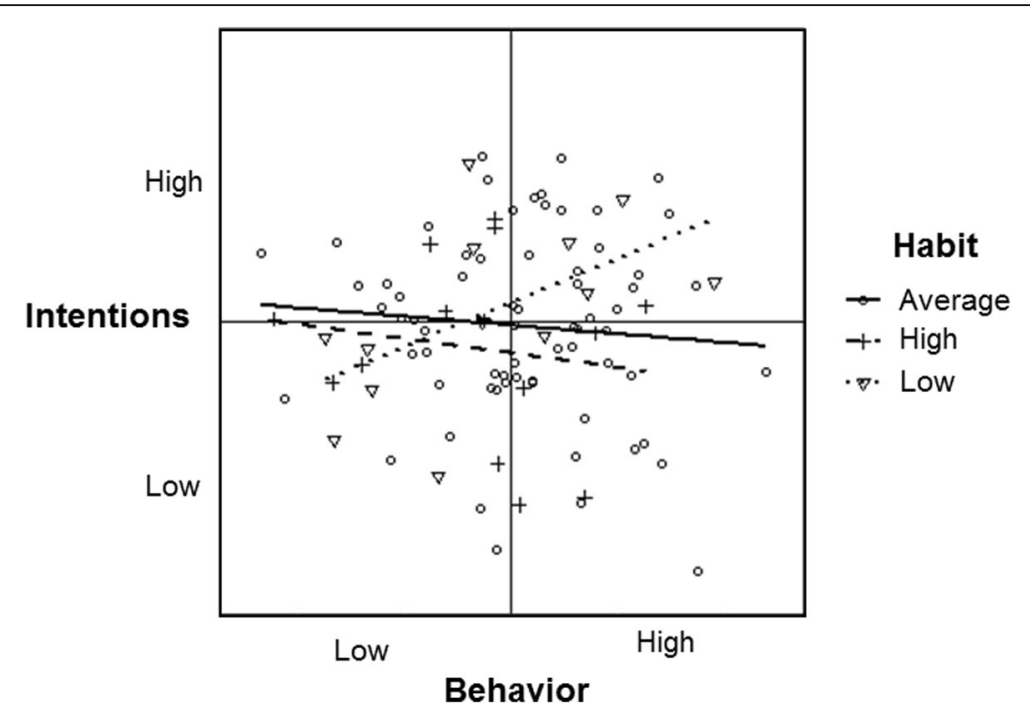

Fig. 4 Habit moderation of the simulated normally distributed and unrelated intention and behavior data (all variables near Gaussian; r's 0.0)

scores), so the intention-behavior association for these cases is restricted into the upper two quadrants. In contrast, the cases with low habit are also distributed on one side of the bottom half of the plot (which represents people with low intention and behavior scores), allowing for a less restricted range of association.

\section{Perceived behavioral control as moderator of intention- behavior associations}

Figure 7 shows the moderation effect of the simulated perceived behavioral control variable on the intentionbehavior data $(b=0.70)$. Of the 7500 simulations, $50 \%$ of moderation effects fell within the range of $b=0.26$ and
1.77. For cases with high perceived control scores, the association between intention and behavior is $r=0.63$ (dashed line), which is nearly twice as strong as it is for those with average perceived control scores $(r=0.34$; solid line). However, as a result of the asymmetry of the intention-behavior data, those with low perceived behavioral control scores also have a strong intentionbehavior association at $r=0.58$ (dotted line). This effect demonstrates that the risk for overextrapolation of intention-behavior moderation effects to those seldomly represented within the data (i.e., those with high behavior but low perceived behavioral control scores)

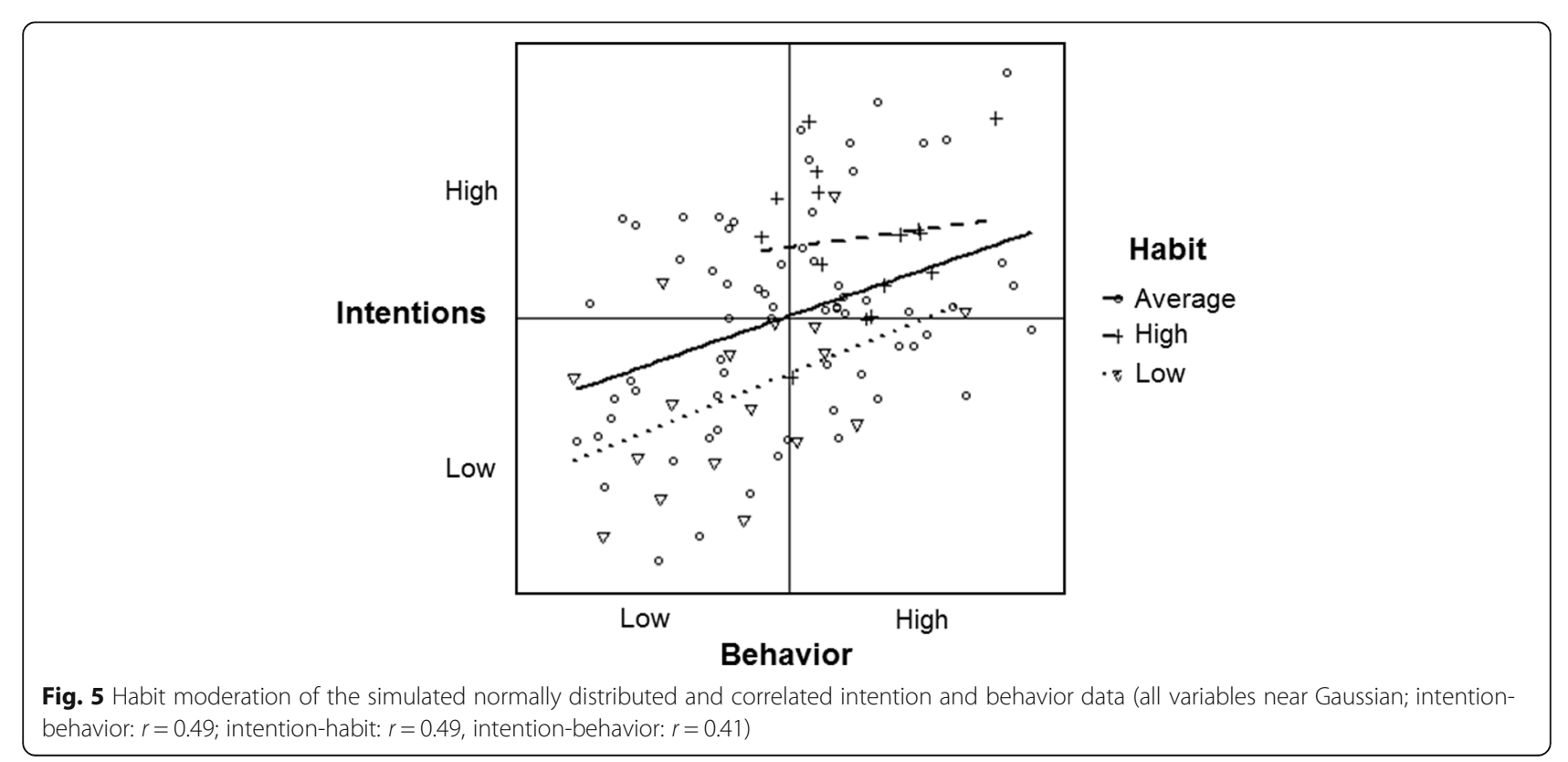




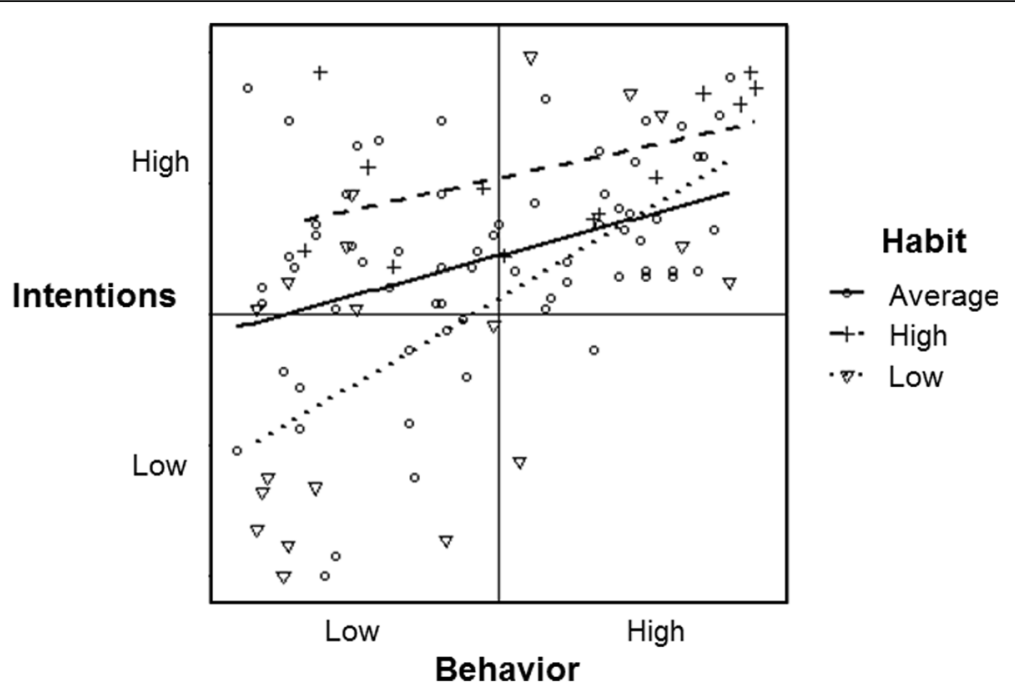

Fig. 6 Habit moderation of the intention and behavior data simulated based on realistic distributions and correlations (intention negatively skewed, behavior positively skewed; intention-behavior: $r=0.49$; intention-habit: $r=0.49$, habit-behavior: $r=0.41$ )

generalizes beyond habit to other moderator variables, as a result of asymmetrical intention-behavior associations.

\section{Discussion}

The asymmetry of intention-behavior relationships is well-documented in physical activity research; far more people fail to act on strong intentions than are frequently physically active despite weak intentions $[15,19]$. Our study provides an innovative demonstration of how this asymmetry can lead to potential misinterpretation of intention-behavior associations and investigations of moderators of these associations. We used simulated data based on normal distributions with no shared variance, correlated parameters with normal distribution, and realistically correlated and non-normally distributed parameters. Comparing the three scenarios, we illustrated how the same correlation coefficient may mask different types of intention-behavior associations. Specifically, we showed that the typical patterns of intention and behavior data in physical activity research leads to violations of two fundamental assumptions of linear modelling. These findings highlight a risk of misinterpreting testing of moderation of the intention-behavior gap for physical activity.

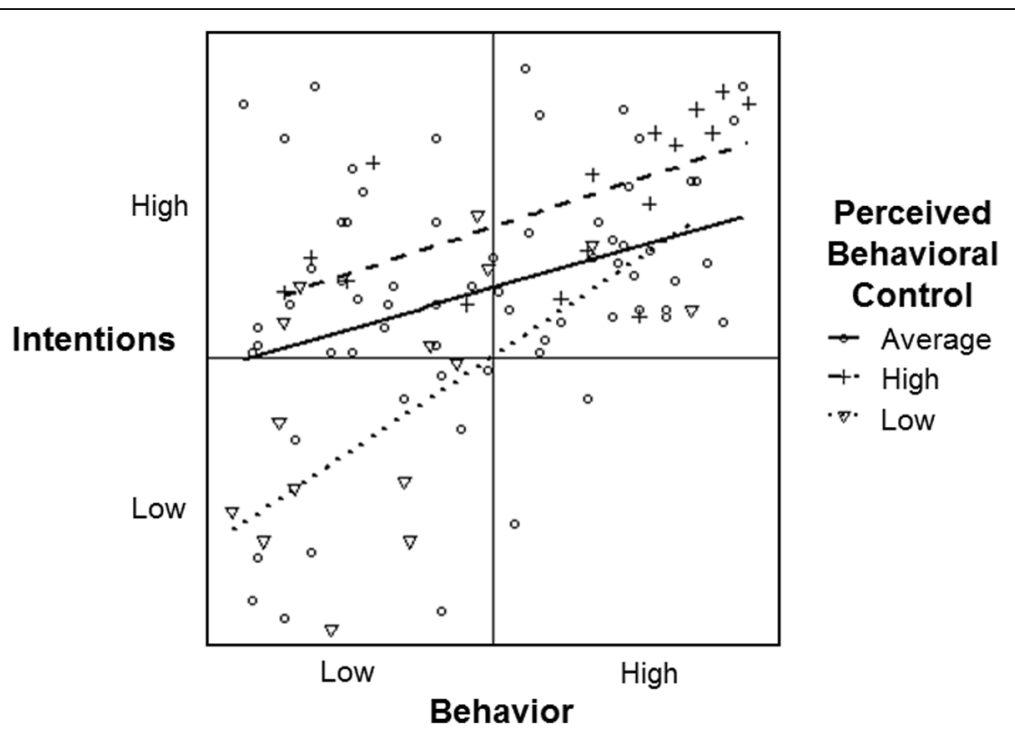

Fig. 7 Perceived behavioral control moderation of the intention and behavior data simulated based on realistic distributions and correlations (intention negatively skewed, behavior positively skewed; intention-behavior: $r=0.49$; intention-perceived control: $r=0.47$, perceived control-behavior: $r=0.33$ ) 
The prevalent risk of misinterpretation of intentionbehavior associations (and moderation thereof) may impact on the implementation of physical activity behavior change interventions. The public health benefits of regular physical activity are numerous and most interventions focus on intention-enhancing strategies such as education, risk awareness, goal-setting and selfmonitoring [2]. If the science underpinning our understanding of how intentions translate into behavior is misinformed, then we may be targeting the wrong behavior change strategies (e.g., intention formation instead of action control) or tailoring our efforts based on a non-existent population sub-group (e.g., those with low intention but high behavioral engagement).

The standard assessment of moderation of the intention-behavior gap is reliant on correlation coefficients of linear regression, which do not capture the intention-behavior profiles that underpin the intentionbehavior gap. If effects were applicable to the entire available data range, the gap would be made of a near equal ratio of people who were intending to engage in the behavior and subsequently did not, and those with no intention who nonetheless engaged in the behavior. Yet this is not usually the case, at least for typical physical activity intention-behavior studies; the stability of the intention-behavior correlation is mostly the result of nonintenders doing nothing. Even if people intended to act, there is still nearly the same probability of the behavior being enacted as winning a coin toss (46\%) [34]. This is interesting because, while it partly supports theories of intention [7, 9-11], it is not helpful for promoting physical activity. This additional information about the reality of intention-behavior associations supports action control theories $[19,47]$, by suggesting intention is necessary but not always sufficient for physical activity.

After illustrating how typical physical activity intentionbehavior data deviate from linear associations with normally distributed residuals, we demonstrated how this asymmetrical association restricts the range of data available for estimating the extent to which a moderator may impact intention-behavior relationships, particularly if the moderator is associated with intention or behavior [28]. Under these circumstances, significant moderation correlation coefficients should not be interpreted as generalizable to people who engage in physical activity without intention, because those people seldom exist.

The commonly tested moderation of habit on the intention-behavior gap in physical activity research is an example of such a circumstance. Theory predicts that habit will override the effect of intention on behavior [6], an effect that has been found in many previous studies [22, 37]. From a theoretical perspective, this should reflect that people with strong habits unintentionally engage in physical activity, because regulation is shifted from the conscious processes needed for intentional action to the automatic cue-to-action processes that elicit habitual responses [48]. This assumption has generated much interest in habit formation interventions as a potential means to behavior maintenance [49, 50]. Commentators have reasoned that, because habit overrides intention, people should be encouraged to make any new physical activity regimens habitual, because they will likely sustain such activity in the face of typical losses in motivation over time [51]. Yet, the evidence base on which this is assumption is based, which appears to show that the effect of intention on behavior weakens as habit strength increases, may be based on statistical byproducts of unmet model assumptions [52]. In such studies, there are typically few people with strong habit but weak intention and almost nobody appears to engage in unintended physical activity behavior. This refutes the idea that those with habits act without intention. This issue was pointed out by Rhodes et al. in their tests of the habit, intention, and physical activity relationship using linear regression compared to a comparison of people categorized based on whether they achieved their intention or not (i.e. which quadrant of the scatterplot their data point fell within) [52]. What they found, and subsequently replicated several times [34], is that, among people with stronger intentions, habit helps to translate intentions into action (presumably by minimizing demands on memory and other attentional resources) [6]. This is perhaps unsurprising: realistically, habit likely forms on the basis of consistent repetition of intentional action [38], such that habits and intentions often correspond [53]. A more nuanced perspective on the practical value of habit formation for behavior maintenance emerges: habit may assist in driving physical activity when people with strong intentions experience dips in motivation (e.g., a habitual distance runner in bad weather conditions). Habit is, however, unlikely to facilitate engagement in activity among people with no intention to be active, or those who strongly intend not to be active. This may explain why, contrary to theory, some research into longer-term effects of habit-formation interventions has observed declines in the focal behavior over time, despite apparent gains in habit strength [54].

Our data should not, however, be taken to indicate that habit never overrides intentional tendencies. Although our data were simulated to reflect the typical concurrence of habit and intentions in the physical activity domain, there are nonetheless valid real-world instances in which habits conflict with intentions. For example, people often form intentions to tackle their bad eating or smoking habits [55-57]. Such instances of discordance between intentions and habits may offer more credible settings for estimating the moderating 
impact of habit on intention-behavior relationships. Although more studies of counter-habitual intentions are needed, it is however notable that the few such studies to date have found little evidence to suggest that habit moderates the intention-behavior gap [35, 55, 56, 58].

We also demonstrated that the same misinterpretation risk generalizes to variables other than habit by showing similar findings using perceived behavioral control. Whereas theory postulates that higher perceived control translates into more effective action control [7], our findings show how these significant moderation effects may be statistical byproducts of asymmetrical intentionbehavior relationships. Other moderators of the intentionbehavior gap in physical activity likely also will be impacted in the same manner. For example, implementation intentions - specific actionable plans about what, where, how, and when intentions will be implemented - are oftentimes proposed as a mechanism by which intentions translate into behavior [59-61]. Given that amongst these studies, implementation intentions and intentions are oftentimes strongly associated, such moderation analysis is susceptible also to misleading conclusions from these unmet assumptions of linear modelling.

\section{Recommendations for future intention-behavior research}

We have some recommendations for how future intention-behavior research could check whether their intention-behavior data are asymmetrical and for managing the imposed risk for misinterpretation of the intention-behavior gap and tests of moderation. These limitations presented here only apply to asymmetrical intention-behavior relationships resulting from non-normal distributions so will not be applicable across all scenarios.

During study development, simple adjustments to recruitment strategy and measurement may make these violations of linearity assumptions less likely. Common physical activity study recruitment methods are prone to oversampling those with more positive activity intentions (e.g., flyers placed in gyms, or recruitment of students enrolled on movement-based university courses). Researchers should engage in recruitment efforts less targeted toward those with strong activity intentions, to increase the likelihood of more normally distributed intention data. Additionally, ensuring an intention measure provides a reasonably broad range of possible response options may reduce the risk of ceiling effect [62]. For example, our experiences of piloting intention measures show that, instead of asking whether people will engage in a set amount of physical activity, more normally-distributed intention data can result from using an open response item requiring respondents to report how much time they intend to be active in a set period. Consideration for the measurement of potential confounders and moderators of intention-behavior associations is also important. For example, some self-report measures of habit strength include items assessing behavioral frequency, which will result in shared variability between habit and behavior variables [36], therefore exacerbating the risk of asymmetrical relationships. If the aim is to investigate the moderating role of habit in intention-behavior associations, alternative measures which do not include the behavioral frequency items may be more applicable $[35,63]$.

Following data collection, efforts can also be made to reduce risks of misleading findings. Assumption testing is essential to ensure the estimates from analyses are interpreted correctly [27-29]. The most prudent assumption testing method harkens back to introductory statistics courses: plot the data. By visualizing the correlation, one can assess whether either intention or behavior data deviates from the normal distribution curve as well as whether there is asymmetry in how the data are dispersed across each of the four quadrants. Unfortunately, past evidence shows that the pattern of asymmetry simulated here is common for intention-behavior associations amidst physical activity research [34]. Fortunately, there are simple ways to address the commonly unmet assumptions of homoscedasticity and linearity.

If the intention-behavior association does seem asymmetrical, it may be tempting to just transform the nonnormal variable (e.g., if physical activity is positively skewed, many people square root or log transform it). Although this may adjust for the statistical modelling violation, it leads to uncertainty when it comes to making meaningful conclusions from the finding. Univariate transformations do not account for the practical problem that we may be generalizing findings to non-existent people (e.g., people who engage in physical activity without reporting intention). There are many more appropriate techniques for managing these assumption violations; which technique is most applicable depends on the research aims, design, and measurement factors.

When the study aim is to investigate decisional intentions (and the measurement reflects that), an option for testing intention-behavior associations and moderators may include profile analyses $[15,19,64]$. This method involves categorizing cases based on the $2 \times 2$ matrix (analogous to the four quadrants in the scatterplots) based on 1 - the decisional intention to engage in the behavior or not, and 2 - whether they subsequently engaged in the intended amount of the behavior or not. The matrix with these category labels from Rhodes and de Bruijn [19] and Sheeran [15] are presented in Table 2. Upon categorizing people into these profiles, the nominal group variable (of which there are four categories) can then be entered as a predictor or outcome in any applicable model. For example, one may test whether habit 
Table 2 The $2 \times 2$ Matrix of Profile Analysis Based on Decisional Intentions and Subsequent Behavior

\begin{tabular}{|c|c|c|}
\hline \multirow[b]{2}{*}{ Did they intend to engage in the set amount of behavior? } & \multicolumn{2}{|c|}{ Did they engage in the intended amount of behavior? } \\
\hline & No & Yes \\
\hline No & $\begin{array}{l}\text { - Non-intenders } \\
\text { - Disinclined abstainers }\end{array}$ & $\begin{array}{l}\text { - Non-intenders exceeding intentions } \\
\text { - Disinclined actors }\end{array}$ \\
\hline Yes & $\begin{array}{l}\text { - Unsuccessful intenders } \\
\text { - Inclined abstainers }\end{array}$ & $\begin{array}{l}\text { - Successful intenders } \\
\text { - Inclined actors }\end{array}$ \\
\hline
\end{tabular}

predicts peoples' intention-behavior profiles. This option may be particularly relevant for health behaviors for which there are evidence-based guidelines around which a dichotomy might be constructed, such as physical activity (i.e. whether $150 \mathrm{~min}$ per week of moderate-tovigorous activity is achieved).

Notably, it can be difficult to identify appropriate cut-off values that are required by profile analyses. Additionally, by categorizing continuous measurements, profile analyses can limit precision and sensitivity $[65,66]$. Given its limitations, we do not recommend the replacement of intention-behavior moderator testing using continuous variables with profile analysis; rather, we recommend using profile analysis to test the generalizability of conclusions drawn from linear moderation estimation. Concordant findings across both analyses would provide more certainty of the meaningfulness and generalizability of the findings. However, if the findings were discrepant, it may indicate the linear moderation is only representative of a certain sub-group of the sample, which could be ascertained via profile analysis.

If supplemental profile analysis is not well-suited for the research question, nonlinear regression may be more suitable for testing intention-behavior associations and potential moderators of the association. This can be applied with the nlstools in $R[67,68]$. The obvious benefit of nonlinear modelling is that it is not reliant on the assumptions underpinning linear modelling, although it is worth noting that nonlinear regression is dependent on its own set of assumptions being met, so the appropriate diagnostics are necessary beforehand. Additionally, it should be noted that sometimes physical activity behavior (and even intention) data take the form of frequency counts (e.g., number of bouts per week/day), in which case the most appropriate analysis strategy would be to account for the Poisson distribution(s) with Poisson (for overdispersion) or negative binomial (for underdispersion) analyses [69]. Given the asymmetry common of health behavior intention-behavior relationships, future research may consider establishing the trajectory of intention-behavior associations and modelling it appropriately (e.g., by incorporating exponential term in regression).

\section{Conclusions}

Advancements in our understanding about what motivates people to engage in physical activity is essential in the global effort to reduce the costly burden of global inactivity [2]. Understanding psychological determinants of physical activity behavior requires understanding not only people's intentions, but also the factors that affect the likelihood of them acting on their intent. The emerging evidence on the intention-behavior gap and its moderating factors is promising, but this study illustrated how the typical patterns of intention and behavior data in physical activity research leads to violations of fundamental assumptions of linear modelling. As a result of these violated statistical assumptions, there is a risk that we are misinterpreting findings important for developing effective physical activity promotion efforts. For example, we have demonstrated that the hypothesis that the effect of intention on behavior weakens as habit strength increases may be based on statistical byproducts of unmet model assumptions. The generalizability of these findings was supported through the replication of the findings with the test of the moderating effect of perceived behavioral control on intention-behavior relationships. To ensure research is practically relevant at a behavioral medicine level, research of intention-behavior associations and moderation of the intention-behavior gap need to be considerate of the risk for misinterpretation from the asymmetry of the real-world phenomenon of intention-behavior associations.

\section{Acknowledgments}

Not applicable.

\section{Consent of publication}

Not applicable.

\section{Authors' contributions}

All authors made substantial contribution to the conception and design of the project. ALR conducted the data simulation and analyses. All authors took part in interpretation of the data, substantial revision of the manuscript, and have read and approved the final manuscript.

\section{Funding}

ALR is supported by funds from the National Health and Medical Research Council of Australia. RER is supported by funds from the Canadian Cancer Society, the Social Sciences and Humanities Research Council of Canada, the Heart and Stroke Foundation of Canada and the Canadian Institutes for Health Research. No funding body had a role in the design, implementation, analysis, or interpretation of the data or in writing the manuscript. 


\section{Ethics approval and consent to participate}

Not applicable.

\section{Competing interests}

The authors declare that they have no competing interests.

\section{Author details}

'School of Health, Medical, and Applied Sciences, Central Queensland University, Building 18, Room 1.33, Bruce Highway, Rockhampton, Queensland 4703, Australia. ${ }^{2}$ School of Exercise Science, Physical and Health Education, University of Victoria, Room 189, McKinnon Building, Victoria, BC V8W 3H5, Canada. ${ }^{3}$ Department of Psychology, Institute of Psychiatry, Psychology, and Neuroscience, King's College London, AH 2.11, Addison House, Guy's Campus, London SE1 1UL, UK.

\section{Received: 2 March 2019 Accepted: 31 July 2019}

Published online: 22 August 2019

\section{References}

1. World Health Organization. Physical inactivity. Geneva: World Health Organization; 2018.

2. World Health Organization. Global strategy on diet, physical activity and health. Geneva: World Health Organization; 2004

3. Australian Bureau of Statistics. Australian health survey: physical activity, 2011-12. Canberra: Australian Institute of Health and Welfare; 2013.

4. Centers for Disease Control and Prevention. Physical activity. Atlanta: Department of Health \& Human Services; 2014.

5. Rhodes RE, Rebar AL. Conceptualizing and defining the intention construct for future physical activity research. Exerc Sport Sci Rev. 2017;45:209-16.

6. Triandis HC. Values, attitudes, and interpersonal behavior. In: Nebraska symposium on motivation. Lincoln: University of Nebraska Press; 1980. p. 195-259.

7. Ajzen I. The theory of planned behavior. Organ Behav Hum Decis Process. 1991;50:179-211.

8. Prochaska J, DiClemente C. Stages and processes of self-change in smoking: toward an integrative model of change. J Consult Clin Psychol. 1983;5:390-5.

9. Rogers RW. Cognitive and physiological processes in fear appeals and attitude change: a revised theory of protection motivation. In: Cacioppo BL, Petty LL, editors. Social psychophysiology: a sourcebook. London: Guilford Press; 1983. p. 153-76.

10. Schwarzer R, Lippke S, Luszczynska A. Mechanisms of health behavior change in persons with chronic illness or disability: the health action process approach (HAPA). Rehabil Psychol. 2011;56:161-70.

11. Bandura A. Social cognitive theory: an agentic perspective. Annu Rev Psychol. 2001;52:1-26.

12. Cooke R, Dahdah M, Norman P, French DP. How well does the theory of planned behaviour predict alcohol consumption? A systematic review and meta-analysis. Health Psychol Rev. 2016:10:148-67.

13. McEachan RRC, Taylor N, Harrison R, Lawton R, Gardner P, Conner M. Metaanalysis of the reasoned action approach (RAA) to understanding health behaviors. Ann Behav Med. 2016;50:592-612.

14. McEachan RRC, Conner M, Taylor NJ, Lawton RJ. Prospective prediction of health-related behaviours with the theory of planned behaviour: a metaanalysis. Health Psychol Rev. 2011;5:97-144.

15. Sheeran P. Intention-behavior relations: a conceptual and empirical review. Eur Rev Soc Psychol. 2002;12:1-36.

16. Rhodes RE, Dickau L. Experimental evidence for the intention-behaviour relationship in the physical activity domain: a meta-analysis. Health Psychol. 2012;31:724-7.

17. Webb TL, Sheeran P. Does changing behavioral intentions engender behavior change? A meta-analysis of the experimental evidence. Psychol Bull. 2006;132:249-68.

18. Sheeran P, Webb TL. The intention-behavior gap. Social Personal Psychol Compass. 2016:10:503-18.

19. Rhodes RE, de Bruijn G-J. What predicts intention-behavior discordance? A review of the action control framework: Exerc Sport Sci Rev. 2013:41:201-7.

20. Sheeran $P$, Orbell S, Trafimow D. Does the temporal stability of behavioral intentions moderate intention-behavior and past behavior-future behavior relations? Personal Soc Psychol Bull. 1999;25:724-34.

21. Godin G, Conner M, Sheeran P. Bridging the intention-behaviour "gap": the role of moral norm. Brit J Soc Psychol. 2005;44:497-512.
22. Gardner B, de Bruijn G-J, Lally P. A systematic review and meta-analysis of applications of the self-report habit index to nutrition and physical activity behaviours. Ann Behav Med. 2011;42:174-87.

23. Rebar AL, Elavsky S, Maher JP, Doerksen SE, Conroy DE. Habits predict physical activity on days when intentions are weak. J Sport Exerc Psychol. 2014:36:157-65.

24. Lippke S, Wiedemann AU, Ziegelmann JP, Reuter T, Schwarzer R. Selfefficacy moderates the mediation of intentions into behavior via plans. Am J Health Behav. 2009:33.521-9.

25. Norman $\mathrm{P}$, Conner M. The theory of planned behavior and exercise: evidence for the mediating and moderating roles of planning on intentionbehavior relationships. J Sport Exerc Psychol. 2005;27:488-504.

26. Mullan B, Wong C, Allom V, Pack SL. The role of executive function in bridging the intention-behaviour gap for binge-drinking in university students. Addict Behav. 2011;36:1023-6.

27. Belsley DA, Kuh K, Welsch RE. Regression diagnostics. New York: Wiley; 1980

28. Fox J. Applied regression analysis and generalized linear models. 2nd ed. New York: Sage; 2008

29. Weisberg S. Applied linear regression. 3rd ed. New York: Wiley; 2005.

30. Altmann J. Observational study of behavior: sampling methods. Behav. 1974:49:227-66

31. Dilorio CK. Measurement in health behavior: methods for research and evaluation Vol. 1. San Fransisco: Wiley; 2006.

32. Gardner B. A review and analysis of the use of 'habit'in understanding predicting and influencing health-related behaviour. Health Psychol Rev. 2015;9:277-95.

33. Rebar AL, Gardner B, Verplanken B. Habit in exercise behavior. In Tenenbaum G, Eklund RC, editors. Handbook of sport psychology. Vol. 4. Wiley: Hoboken; 2018

34. Rhodes RE, de Bruijn G-J. How big is the physical activity intentionbehaviour gap? A meta-analysis using the action control framework. Brit J Health Psychol. 2013:18:296-309.

35. Gardner B, Abraham C, Lally P, de Bruijn G-J. Towards parsimony in habit measurement: testing the convergent and predictive validity of an automaticity subscale of the self-report habit index. Int J Behav Nutr Phys Act. 2012:9:102-13.

36. Verplanken B, Orbell S. Reflections on past behavior: a self-report index of habit strength. J Appl Soc Psychol. 2003:33:1313-30.

37. Rebar AL, Dimmock JA, Jackson B, Rhodes RE, Kates A, Starling J, Vandelanotte C. A systematic review of non-conscious regulatory processes and physical activity. Health Psychol Rev. 2016;10:395-407.

38. Lally P, Van Jaarsveld CH, Potts HW, Wardle J. How are habits formed: modelling habit formation in the real world. Euro J Soc Psychol. 2010;40: 998-1009.

39. Rhodes RE, Dickau L. Moderators of the intention-behaviour relationship in the physical activity domain: a systematic review. Brit J Sport Med. 2013;47: 215-25.

40. Morris TP, White IR, Crowther MJ. Using simulation studies to evaluate statistical methods. Stat Med. 2017. https://doi.org/10.1002/sim.8086.

41. Simmons JP, Nelson LD, Simonsohn U. False-positive psychology: undisclosed flexibility in data collection and analysis allows presenting anything as significant. Psychol Sci. 2011;22:1359-66.

42. R Core Team. R: A language and environment for statistical computing. Vienna, Austria: R Foundation for Statistical Computing. 2017. https://www.rproject.org/.

43. Venables WN, Ripley BD. Modern applied statistics with S. 4th ed. New York Springer; 2002.

44. Mundford DJ, Schaffer J, Kim M-J, Shaw D, Thongteeraparp A. Number of replications required in Monte Carlo simulation studies: a synthesis of four studies. J Mod Appl Stat Methods. 2011;10:19-28.

45. Peng RD. simpleboot: Simple bootstrap routines. R package version 1.1-3; 2008

46. Hagger MS, Chatzisarantis NL, Biddle SJ. A meta-analytic review of the theories of reasoned action and planned behavior in physical activity: predictive validity and the contribution of additional variables. J Sport Exerc Psychol. 2002;24:3-32

47. Kuhl J Volitional aspects of achievement motivation and learned helplessness: toward a comprehensive theory of action control. In: Maher BA, Maher WA, editors. Progress in experimental personality research. Vol. 13. New York: Academic Press; 1984. p. 99-171.

48. Lally P, Wardle J, Gardner B. Experiences of habit formation: a qualitative study. Psychol Health Med. 2011;16:484-9. 
49. Rothman AJ, Sheeran P, Wood W. Reflective and automatic processes in the initiation and maintenance of dietary change. Ann Behav Med. 2009;38:s4-s17.

50. Verplanken B, Wood W. Interventions to break and create consumer habits. J Public Policy Mark. 2006;25:90-103.

51. Hagger MS. Habit and physical activity: theoretical advances, practical implications, and agenda for future research. Psychol Sport Exerc. 2019;42: 118-29.

52. Rhodes RE, de Bruijn G-J, Matheson DH. Habit in the physical activity domain: integration with intention temporal stability and action control. J Sport Exerc Psychol. 2010;32:84-98.

53. Ouellette JA, Wood W. Habit and intention in everyday life: the multiple processes by which past behavior predicts future behavior. Psychol Bull. 1998;124:54-74.

54. Judah G, Gardner B, Aunger R. Forming a flossing habit: an exporatory study of the psychological determinants of habit formation. B Journal Health Psychol. 2012;18:338-53.

55. Gardner B, Corbridge S, McGowan L. Do habits always override intentions? Pitting unhealthy snacking habits against snack-avoidance intentions. BMC Psychol. 2015;3:8-16.

56. Verplanken B, Faes S. Good intentions, bad habits, and effects of forming implementation intentions on healthy eating. Euro J Soc Psychol. 1999;29: 591-604.

57. Orbell S, Verplanken B. The automatic component of habit in health behavior: habit as cue-contingent automaticity. Health Psychol. 2010;29:374-83.

58. Murtagh S, Rowe DA, Elliott MA, McMinn D, Nelson NM. Predicting active school travel: the role of planned behavior and habit strength. Int J Behav Nutr Phys Act. 2012;9:65-74.

59. Ajzen I, Czasch C, Flood MG. From intentions to behavior: implementation intention, commitment, and conscientiousness. J Appl Soc Psychol. 2009;39: 1356-72.

60. Fennis BM, Adriaanse MA, Stroebe W, Pol B. Bridging the intention-behavior gap: inducing implementation intentions through persuasive appeals. J Consum Psychol. 2011;21:302-11.

61. Gollwitzer PM, Sheeran P. Implementation intentions and goal achievement: a meta-analysis of effects and processes. Adv Exp Soc Psychol. 2006;38:69-119.

62. Cramer D, Howitt DL. Ceiling effect. In: Cramer D, Howitt DL, editors. The SAGE dictionary of statistics: A practical resource for students in the social sciences. London: SAGE; 2010. p. 21.

63. Rebar AL, Rhodes RE, Gardner B, Verplanken B. The measurement of habit In: Verplanken B, editor. The psychology of habit. New York: Springer; 2018. p. 31-49.

64. Godin G, Conner M. Intention-behavior relationship based on epidemiologic indices: an application to physical activity. Am J Health Promo. 2008;22:180-2.

65. Bennette C, Vickers A. Against quantiles: categorization of continuous variables in epidemiologic research, and its discontents. BMC Med Res Methodol. 2012;12:21-5

66. van Walraven C, Hart RG. Leave 'em alone-why continuous variables should be analyzed as such. Neuroepidemiology. 2008;30:138-9.

67. Baty F, Ritz C, Charles S, Brutsche M, Flandrois J-P, Delignette-Muller M-L. A toolbox for nonlinear regression in R: the package nlstools. J Stat Softw. 2015;66:1-21.

68. Cortina JM. Interaction, nonlinearity, and multicollinearity: implications for multiple regression. J Manag. 1993;19:915-22.

69. Alexander N. Review: analysis of parasite and other skewed counts. Tropical Med Int Health. 2012;17:684-93.

\section{Publisher's Note}

Springer Nature remains neutral with regard to jurisdictional claims in published maps and institutional affiliations.

Ready to submit your research? Choose BMC and benefit from:

- fast, convenient online submission

- thorough peer review by experienced researchers in your field

- rapid publication on acceptance

- support for research data, including large and complex data types

- gold Open Access which fosters wider collaboration and increased citations

- maximum visibility for your research: over $100 \mathrm{M}$ website views per year

At BMC, research is always in progress.

Learn more biomedcentral.com/submissions 